\title{
The Placebo Standard in Regulatory Market Approval: Why the Declaration of Helsinki Has Been Ignored for 35 years
}

\author{
Patrick James Villeneuve*†, B.Sc., B.A.Sc.
}

\section{INTRODUCTION}

The Declaration of Helsinki, a landmark document promoting ethical clinical research, was adopted by the $18^{\text {th }}$ World Medical Assembly in June 1964. Among the many issues dealt with, the declaration specifically states that:

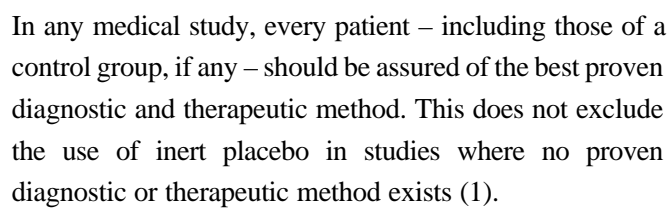

The Declaration of Helsinki has been reaffirmed at subsequent assemblies of the World Medical Assembly, the most recent being the $48^{\text {th }}$ General Assembly of the World Medical Association in 1996. In the period from 1964 to the present, most clinical trials employed a placebo control arm, and most drugs approved for sale were evaluated on the basis of one or more placebocontrolled studies. Why has there been little movement in restricting and limiting the role of placebo in clinical trials? The ideological conflict that currently exists between ethicists and regulatory agencies needs to be resolved before the Declaration of Helsinki can ensure the best proven treatment for all patients enrolled in clinical trials.

The basics of new drug approval will be presented, followed by a discussion of the ethical underpinnings of

\footnotetext{
* To whom correspondence should be addressed: 3655 Promenade Sir William Osler, Montreal, QC, H3G 1Y6. E-mail: ville026@med.mcgill.ca.

$\dagger$ Faculty of Medicine, McGill University.
}

clinical trials in general. An examination of some recent studies employing placebo controls will then be undertaken. Statistical considerations surrounding the use of placebo-controlled studies and some alternatives will also be discussed.

\section{REGULATORY APPROVAL OF PHARMA- CEUTICALS}

New pharmaceuticals must pass several stages of development prior to receiving approval for marketing. Drug studies represent step-wise progressions which use data in early small-scale studies to support larger, more definitive investigations (2). These are divided by time into four main areas of investigation, referred to as phases I - IV. Phase I studies are generally performed early in drug development and are primarily to determine short-term safety and pharmacologic parameters in human subjects. Prior to phase I studies, investigations involve animal and in vitro models. Phase II studies begin to explore the use of the drug as therapy, while later (and larger) phase III studies seek to confirm the efficacy of the drug as therapy. Phase IV studies are undertaken once regulatory approval has been granted for a variety of reasons, including optimization of drug use or further characterization of drug-drug interactions, as examples.

\section{ARE PLACEBO CONTROLS ETHICAL?}

Benjamin Freedman summarized the requirements of ethical clinical research in his concept of 'clinical equipoise' (3). Clinical equipoise represents a genuine state of uncertainty within the professional community with respect to the merits of each arm of a trial. In more concrete terms, clinical equipoise exists if it is truly 
unknown whether treatment $\mathrm{A}$ is superior to treatment B for a given medical condition (3).

Trials find ethical justification under the premise of clinical equipoise. Thus, when expert opinion is divided over the relative merits of treatment A versus B in a given condition, a clinical trial is necessary to resolve the question of treatment superiority.

A placebo-controlled trial is one where a treatment of interest is compared to an inactive treatment (i.e. placebo). To fulfill the requirements of clinical equipoise, a genuine uncertainty as to the merits of a given treatment versus placebo must exist. Thus, to allow an ethical comparison versus placebo, no other treatment can exist. A trial for any condition for which an established treatment exists violates clinical equipoise if that trial uses placebo as a basis for comparison.

A 1995 review of published trials involving ondansetron, an antiemetic agent, found that of 18 published studies involving 8806 patients, 2620 were randomized to placebo (4). Postoperative nausea and vomiting, the indication tested in the series of studies, already had effective drugs available, among them dexamethasone, droperidol and metoclopramide. Emesis in post-treatment settings is a source of considerable morbidity and discomfort, especially in oncology. The fact that almost a third of study subjects in trials involving postoperative nausea and vomiting were randomized to placebo in the face of effective antiemetic treatment is ethically questionable. The rationale provided by many investigators was the following: The standard of care postoperatively does not routinely include antiemetic treatment and, therefore, placebo control arms were providing standard care. To refute this claim, one must realize that if antiemetic treatment was not the standard of care in routine postoperative management, emesis in the postoperative setting is not a pressing concern. A clinical trial in this setting cannot be motivated by clinical equipoise, as there is no question as to the superiority of a given therapy. This basic consideration aside, in cases where there is significant patient morbidity, as is the case in nausea and vomiting, the use of placebo where effective treatment exists is unethical. Investigators attempt to address such ethical considerations with the use of 'rescue medication' representing effective anti-emetic treatment offered to all subjects within a trial who have significant nausea after a predetermined interval of time despite the administration of a test drug (be it placebo or the particular test compound). While the use of rescue medications partially alleviates the ethical dilemma of randomizing subjects to placebo control arms of the study, it seems to predicate a certain expectation as to the outcome of the randomization. A priori expectations of outcomes are not consistent with the concept of clinical equipoise.

Comparisons of test drugs to placebo in the face of effective therapies have been described in trials of antihelminthic agents, disease-modifying anti-rheumatoid arthritis drugs, antidepressants and treatments for congestive heart failure and hypertension (5). Each of these diseases carries significant morbidity and longterm sequelae if left untreated - and yet are compared to placebo in lieu of the currently accepted treatments.

\section{WHY ARE PLACEBO-CONTROLLED STUDIES STILL THE BASIS OF DRUG APPROVAL?}

Placebo-controlled studies continue to be endorsed by regulatory bodies in the interests of accuracy. When a placebo is not used as the comparison, difficulties of statistical noise dominate, making determinations of efficacy challenging. It is also recognized by those same regulatory bodies, that "placebo-controlled trials, whatever their advantages in interpretability, are obviously not ethically acceptable where existing treatment is life-prolonging" (6). Unfortunately, this statement does not address the issue of diseases where treatment has significant impact on present and future quality of life.

Pharmaceutical companies, accruing considerable expense in phase I, II and III clinical trials, are reluctant to perform studies which are not going to be favourably reviewed by the regulatory bodies. Since regulatory agencies generally insist on one or more placebo-controlled trials to demonstrate efficacy, the types of studies undertaken by pharmaceutical companies, unsurprisingly, involve placebo-control arms.

The reasoning behind regulatory insistence on placebo controls can be understood as a basic argument of uncertainty. Regulatory bodies are primarily interested in issues of safety and efficacy of a given treatment. The use of placebo can control "for all potential differences on the actual or apparent course of [a] disease other than those arising from the pharmacologic action of the test drug" (7). The current design of clinical trials causes difficulties in the discernment of specific treatment advantages without the use of placebo, as Dr. Temple of the Food and Drug Administration related in 1997:

\footnotetext{
I want to give you a brief anecdote, which I found sobering. This is about a drug called Carvedilol. It's a betablocker with some alpha-blocking components that you take twice a day. The reason you take it twice a day is that in a study done in the United States, it was perfectly clear that taking $25 \mathrm{mg}$, the largest dose you could tolerate once
} 
a day, did not lead to adequate control at the end of the day. And we don't approve drugs that don't give you 24-hour control. They had studied over 8,000 people in comparative trials in Europe, because Europe likes comparative trials, that did not include a placebo. And the dose they studied was $25 \mathrm{mg}$ once a day. They showed in those 8,000 patients, no difference between known effective once a day therapies and their drug, which we know from the U.S. study doesn't work (8).

It can be concluded, therefore, that large sample sizes alone are not adequate to demonstrate efficacy in comparative trials with active-controls. If comparative trials of active treatments are to become acceptable to regulatory agencies, a greater emphasis must be placed on placebo-controlled preclinical dose-ranging studies prior to any large-scale active-control studies.

Recently, active-controlled clinical trials GUSTO for thrombolytics (9) and TOHMS for antihypertensives (10) have been undertaken. These large-scale clinical trials were done "well after approval for marketing" (11) for the purposes of comparing competing treatments. Ideally, the motivation for the GUSTO and TOHMS studies should be applied to the regulatory process. This would allow for regulatory approval to be granted for safe and efficacious treatments, while providing data on relative efficacy.

\section{HOW CAN WE RECONCILE REGULATORY AND ETHICAL CONCERNS?}

The clinician has little interest in a comparison of a treatment versus placebo but, rather, is interested in the merits of treatments A and B for condition X. More specifically, a clinician is concerned with relative efficacy, side effect profiles and issues of toxicity. The latter concerns of side effects and toxicities are best addressed in phase II studies, where a placebo control is crucial due to the necessarily low numbers of patients. The information generated from phase II studies is clinically relevant: Once side effect profiles are generated, a clinician can easily compare between different side-effect profiles. Phase III studies showing clinical efficacy are currently placebo-controlled. The clinician has no information other than ' $\mathrm{A}$ is better than placebo' or 'B is better than placebo'. How can a clinician differentiate between the therapies? Riskbenefit considerations are impossible to ponder in light of the absence of comparative information on efficacy. As clinical trials involve highly selected subsets of patients, it is difficult to extrapolate findings on specific treatments to the general patient population: "It is often less clear how well the drug will work under different conditions and in patients who do not resemble those in the trials" (9).
Meta-analyses are presently emerging as a source of retrospective, comparative information on the relative efficacy of differing treatments for a given condition. While these undoubtedly provide valuable data, there is an absolute reliance on past clinical trials. For current and emerging treatments, clinicians cannot afford to wait for the publication of meta-analyses. Phase III clinical trials must begin to provide such comparative information.

One possible approach involves 'add-on' studies (10), where both treatment arms receive standard therapy, and one of the two arms receives the test drug in addition (i.e. A vs. $\mathrm{A}+\mathrm{B}$ ). Trials of the add-on type are commonplace in AIDS trials and in oncology. The add-on structure could be adapted to all trials where effective treatments exist. An additional advantage of the add-on type is seen in studies where treatments of interest have different mechanisms of action (10). Without dealing specifically with the statistical methodology involved, it is possible to distill treatment effect for the test drug (B) from the total treatment effect $(A+B)(12)$.

In conditions where polytherapy is the norm, the concept of add-on studies clearly shows merit, even when the specific condition treated is not lifethreatening. Direct comparisons (A vs. B) may be more useful to the clinician, however, in conditions where monotherapy is routinely used. A recent meta-analysis showed that in 19 of 52 studies, ondansetron was no better than placebo, and of these studies, the median number of enrolled subjects was only 30 (13). These data demonstrate that inadequate study designs are all too frequently used. If it is difficult to show superiority over placebo in clinical trials, the success rate would presumably drop even further once confounding effects of active comparisons are introduced. To counteract this, one may choose to increase the study size, at which point economic constraints are often invoked. As related above regarding Carvedilol, a large trial does not necessarily imply success (8).

A 1998 meta-analysis of ondansetron and other antiemetics showed that optimal doses of compared drugs were unknown, clearly contributing to the lack of success (only 35\%) in demonstrating treatment advantages (11). Allowing phases I and II to remain placebo-controlled will ensure valid toxicity and doseranging data. The success of active-control studies is dependent on accurate toxicity and dosing information having been established prior to initiation of the activecontrol study.

It has been recommended that once phase II studies are completed, regulatory bodies and sponsoring pharmaceutical companies should jointly fund Phase III studies (3). In this way, studies of adequate size could 
be undertaken to ensure that comparative studies using established treatments generate useful information for both clinicians and regulators. By allowing placebo control in the most sensitive phase II studies, regulatory bodies can be confident in toxicity, side effect and doseranging data, allowing phase III studies to concentrate on relative efficacy with established treatments. In this way, clinical investigators can adhere to the Declaration of Helsinki without risking difficulties when seeking market approval.

\section{CONCLUSION}

There is no question that placebo-controlled studies are unethical under certain circumstances. A conflict arises when one attempts to reconcile concerns of safety and efficacy with issues of ethics. Just as the Declaration of Helsinki recognizes that placebo is required "where no proven diagnostic or therapeutic method exists" (1), ethicists must understand a need for some placebo controls in the early stages of drug development, and regulators must recognize the clinical need for useful comparative information. A fundamental change in the way clinical research is undertaken must occur to allow for a regulatory process which satisfies ideas of clinical equipoise while assuring safe and effective treatments.

\section{REFERENCES}

1. World Medical Association Recommendations Guiding Physicians in Biomedical Research Involving Human Subjects. World Medical Association 48th General Assembly; 1996.
2. ICH Harmonised tripartite guideline: General considerations for clinical trials (E8). Step 4; July 17, 1997

3. Freedman B. Equipoise and the ethics of clinical research. New England Journal of Medicine 1987; 317: 141-145.

4. Aspinall RL, Goodman NW. Denial of effective treatment and poor quality of clinical information in placebo controlled trials of ondansetron for postoperative nausea and vomiting: a review of published trials. BMJ 1995; 331: 844-846.

5. Rothman KJ, Michels KB. The continuing unethical use of placebo controls. New England Journal of Medicine 1994; 331: 394-398.

6. Brody BA. When are placebo-controlled trials no longer appropriate? Controlled Clinical Trials 1997; 18: 602-612.

7. ICH Harmonised tripartite guideline: choice of control group and related issues in clinical trials (E10) Step 4; July 20, 2000 .

8. Temple R. Appears in: The use of placebos in clinical trials and the ethics of the use of placebos. US Department Of Health and Human Services, Food and Drug Administration, Center for Drug Evaluation and Research, Committee for Advanced Scientific Education Seminar Series; 1999.

9. GUSTO Investigators. An international randomized trial comparing four thrombolytic strategies for acute myocardial infarction. New England Journal of Medicine 1993; 329: 673-682.

10. Mascioli SR, Grimm RH Jr, Neaton JD, et al. Characteristics of participants at baseline in the treatment of mild hypertension study (TOHMS). American Journal of Cardiology 1990; 66: 32-50.

11. Henry D, Hill S. Comparing treatments. BMJ 1995; 310: 1279.

12. Collins R. Appears in: Basic statistical considerations for the evaluation of active controlled clinical trials. 82nd meeting of the cardiovascular and renal drugs committee. US Department of Health and Human Services, Public Health Service, Food and Drug Administration; 1997.

13. Tramer MR, Reynolds DJ, Moore RA, McQuay HJ. When placebo controlled trials are essential and equivalence trials are inadequate. BMJ 1998, 317: 875-880.

Patrick James Villeneuve is currently in the third year of his medical studies. He has degrees in both biochemistry and chemical engineering, and has worked in the area of pharmaceutical approval and regulation at Health Canada as a summer student. 